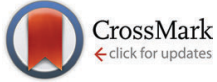

Cite this: Phys. Chem. Chem. Phys., 2015, 17, 27357

Received 13th February 2015, Accepted 14th September 2015

DOI: $10.1039 / c 5 c p 00945 f$

www.rsc.org/pccp

\title{
Adsorption of poly acrylic acid onto the surface of calcite: an experimental and simulation study $\dagger$
}

\author{
David J. Sparks, ${ }^{a}$ Maria E. Romero-González, ${ }^{\star b}$ Elfateh El-Taboni, ${ }^{c}$ \\ Colin L. Freeman, ${ }^{a}$ Shaun A. Hall, ${ }^{a}$ Gabriella Kakonyi, ${ }^{\text {b Linda Swanson, }}{ }^{d}$ \\ Steven A. Banwart ${ }^{\mathrm{b}}$ and John H. Harding ${ }^{{ }^{\mathrm{a}}}$
}

\begin{abstract}
Macromolecular binding to minerals is of great importance in the formation of biofilms, and carboxylate functional groups have been found to play a pivotal role in the functioning of these macromolecules. Here we present both fluorescence time-resolved anisotropy measurements and simulation data on the conformational behaviour and binding of a poly acrylic acid polymer. In solution the polymer exhibits a $\mathrm{pH}$ dependent behaviour, with a coiled conformation at a low $\mathrm{pH}$ and extended conformation at higher $\mathrm{pH}$ values. The polymer is readily adsorbed on the surface of calcite, preferring to bind in an extended conformation, with the strength of the adsorption dependent on the $\mathrm{pH}$ and presence of counter ions. We discuss the reasons why the calculated adsorption free energy differs from that obtained from a Langmuir isotherm analysis, showing that they refer to different quantities. The enhanced binding of the extended conformations shows the importance of flexibility in the binding of macromolecules.
\end{abstract}

\section{Introduction}

The attachment and agglomeration of microbial cells to solid surfaces provides a stable growth environment for the cells, protection from harsh environmental conditions and enhances catalytic functions through the localization of cells into biofilms. ${ }^{1}$ The formation of these biofilms on solid surfaces is of great interest to biotechnology in areas such as medical implant contamination where the formation of biofilms can lead to infections, ${ }^{2}$ the pollution of groundwater supplies, ${ }^{3}$ soil science and bio manufacturing. ${ }^{4}$ The excretion of an extracellular polymeric substance (EPS), composed of macromolecules such as polysaccharides, proteins and lipids promotes the development of the biofilms on mineral surfaces. ${ }^{5}$ Previous studies have shown the importance of functional groups such as carboxylates in the attachment of these macromolecules to such surfaces. ${ }^{6}$ Not only is the substrate important in the selection of macromolecules involved with binding, the attachment of these macromolecules to the mineral surfaces also has a large effect on the mineral

\footnotetext{
${ }^{a}$ Department of Materials Science and Engineering, University of Sheffield, Sir Robert Hadfield Building, Mappin Street, Sheffield, S1 3JD, UK. E-mail: j.harding@sheffield.ac.uk

${ }^{b}$ Department of Civil and Structural Engineering, Kroto Research Institute, University of Sheffield, Broad Lane, Sheffield, S3 7HQ, UK.

E-mail:m.e.romero-gonzalez@sheffield.ac.uk

${ }^{c}$ Department of Chemistry, University of Benghazi, Libya

${ }^{d}$ Department of Chemistry, University of Sheffield, Sheffield, S3 7HF, UK

$\dagger$ Electronic supplementary information (ESI) available: Details of the simulation parameters are available. See DOI: 10.1039/c5cp00945f
}

phase itself. Organic molecules such as proteins (both structured and disordered), ${ }^{7-9}$ peptides, ${ }^{10}$ polysaccharides ${ }^{11}$ and polymers ${ }^{12}$ greatly affect the growth and polymorph selection of, for example, calcium carbonate systems. These biomolecules inhibit or promote the growth of calcite, the most stable $\mathrm{CaCO}_{3}$ polymorph, or promote the formation of other, less stable polymorphs.

The design of the experiments set out in this paper is focused on the importance of carboxylate groups found on macromolecules from bacteria cell walls and EPS secreted by bacteria to promote adhesion, such as lipopolysaccharides and mycolic acid. The well-known polymer of acrylic acid (polyacrylic acid, PAA) can be used as a simple model for these more complex EPS components. ${ }^{6,13,14}$ In this work both experimental fluorescence studies and computer simulations have been employed to analyse the binding of PAA macromolecules to the surface of calcite. Fluorescence time-resolved anisotropy measurements (TRAMS) were used to collect the experimental data used in this study. TRAMS is a powerful technique that monitors the segmental motion of a polymer in solution and at the polymersolid interface. ${ }^{15-17}$ The difficulty with studying large macromolecules in computer simulations is the number of degrees of freedom that the macromolecule has and the large energy barriers between the different conformations that it can adopt. It is difficult to sample all the conformational configurations using straightforward molecular dynamics simulations, and in order to increase the efficiency of sampling this phase space, a different approach must be used. There are several methods available that increase the sampling of a system, such as replica exchange molecular dynamics ${ }^{18}$ (REMD) and umbrella sampling. ${ }^{19}$ 
REMD is a sampling technique that uses duplicate simulations at different temperatures to overcome energy barriers between conformations. Umbrella sampling uses a biased potential to drive a simulation towards certain reaction coordinates. In this work we have utilised the metadynamics method to increase the sampling of the system. This method was developed in 2002 by Laio and Parrinello ${ }^{20}$ and also uses a biased potential to drive a simulation forward. The simulation methodology developed here will be a useful tool in future projects to study the behaviour of other macromolecules, such as polymers and peptides, in solution and on mineral surfaces.

A PAA chain with a number average molar mass of $M_{\mathrm{n}}=$ $19580 \mathrm{~g} \mathrm{~mol}^{-1}$ (272-mers) was used in the experimental work reported here. Due to the difficulty of simulating such a large polymer in molecular dynamics simulations, a short chain, 28-mers of PAA with molecular weight of $c a .2000 \mathrm{~g}$ mol was used in the simulation work. This shorter chain polymer is expected to behave slightly differently to the larger chain polymers. PAA is a well-studied polymer and has been the focus of several experimental and computational studies in the past. Laguecir et $a .^{21}$ studied the behaviour of PAA in solution and found a decrease in the degree of ionisation when increasing the chain length of the polymer, a decrease in the diffusivity of PAA with increasing chain length, and a decrease in diffusivity with increasing $\mathrm{pH}$. Bulo et al. ${ }^{22}$ investigated the interaction between short chain PAA and calcium ions in solution using computational simulations and found that at high ionic strengths the PAA chains exhibit a extended conformation stabilised by calcium ions. The same research group ${ }^{23}$ also performed computational studies on the complexation of PAA with calcium and carbonate ions, showing that PAA chains strongly bind to calcium carbonate species. Computational simulations in vacuum of longer PAA chains by Liu et al. ${ }^{24}$ show the favourable absorption of the polymer onto calcite surfaces due to the interaction between the functional groups and the surface. Aschauer et al. ${ }^{25}$ later showed that the binding of PAA must have a high entropic contribution due to the disruption of the highly ordered water layer on the calcite surface, a conclusion shared by Geffroy et al., ${ }^{26}$ who also showed that small molecular weight polymers will have a greater affinity for the surface than large molecular weight polymers. Our study analyses the adsorption of PAA to calcite surfaces and the conformation of the polymer at varying $\mathrm{pH}$ values, comparing experimental data with simulation results.

\section{Methods}

\subsection{Synthesis and characterisation of calcite and poly acrylic acid}

Deionised double-distilled water of approximate resistivity 18.2 $\mathrm{M} \Omega \mathrm{cm}$ was used in all studies. Calcite crystals $\left(\mathrm{CaCO}_{3}\right)$ were prepared according to the method of Schultz et al. ${ }^{27}$ and had a particle diameter of $32 \mu \mathrm{m}$ with a $\mathrm{pH}$ at the point of zero charge of 8.45 ( $1 \mathrm{wt} \%$ in water). The calcite particles obtained were characterised using Scanning Electron Microscopy (Philips XL-40 SEM), powder XRD and FT-IR (PerkinElmer), BET surface by gas adsorption and particle size distribution by Laser diffraction. The SEM micrograph obtained (Fig. S1, ESI $\dagger$ ) showed that particles are a combination of several nanometer size crystals of a predominantly rhombic face, with some very small crystals of spherical shape. The obtained particles are similar in size and shape to the ones reported. ${ }^{27,28}$ The XRD pattern (Fig. S2, ESI $\dagger$ ) showed the five characteristic peaks for calcite. The peaks fit well when compared with a pure calcite reference (Table S1, ESI $\dagger$ ) indicating the presence of pure calcite with a rhombohedral unit cell as observed from the SEM data. The FT-IR spectra of the synthesised powder showed the characteristic peaks of calcite at $1400 \mathrm{~cm}^{-1}$. No hydroxyl peaks were observed, suggesting that no $\mathrm{Ca}(\mathrm{OH})_{2}$ was present and the calcium ions were converted into calcite. The zeta potential of the calcite surface was measured using a $10 \mathrm{wt} \%$ solution of calcite over a range of $\mathrm{pH}$ values (Zetasizer Malvern Instruments). The $\mathrm{pH}_{\mathrm{pzc}}$ of the calcite produced was 8.85. The net surface of calcite was found to be positive at $\mathrm{pH} 7$ and negative after the $\mathrm{pH}_{\mathrm{pzc}}$. The surface area of the calcite was determined as $3.27 \mathrm{~m}^{2} \mathrm{~g}^{-1}$ and the average particle size obtained was $3.03 \mu \mathrm{m}$ (Fig. S4 and S5 respectively, ESI $\dagger$ ).

Prior to use, 4-amino naphthalene-1-sulfonic acid (AmNS) (Aldrich, 97\%) was purified by extraction with warm hexane. The residue was removed and dried under vacuum over $\mathrm{CaCl}_{2}$ to produce a yield of $c a$. $74 \%$ with a melting point of 300$302{ }^{\circ} \mathrm{C}$, which was stored in a dark coloured bottle in a cold place. Acenaphthylene (ACE) (Lancaster, 90\%) was purified by multiple recrystallisations from methanol, followed by vacuum sublimation. The purified fluorophore (ACE) with a melting point of $86.5-87.0{ }^{\circ} \mathrm{C}$ was stored at $-10{ }^{\circ} \mathrm{C}$. Before use, a monomer of acrylic acid (AA) was vacuum distilled at room temperature to remove the gas phase inhibitors (monomethyl ether hydroquinone) that are included in the supplied material to prevent polymerisation. The distillation was carried out with the distillate flowing into an iced vessel. The purified AA was stored at $-10{ }^{\circ} \mathrm{C}$. Solutions with a monomer to solvent weight ratio of approximately 1:4 were made using 1-4 dioxane as solvent. The monomer solutions were poured into ampoules, then degassed to remove dissolved oxygen using a freezepump-thaw cycle on a vacuum line. Finally the ampoules were placed in a water bath at $60{ }^{\circ} \mathrm{C}$ to polymerise for one day. The reactions were polymerised by a free radical mechanism using azobisisobutyronitrile (AIBN, $1000 \mathrm{mg} \mathrm{L}^{-1}$ ) as initiator. The polymers were purified by multiple dissolutions (three times) and subsequent precipitation using methanol as the solvent and diethyl ether as non-solvent. The polymers produced were then dried in a vacuum oven for at least 24 hours before being weighed. The polymers were labelled with the fluorescent labels AmNS and ACE following a modified procedure described elsewhere. ${ }^{29}$ The products were washed with dichloromethane (Sigma-Aldrich) and the aqueous layers were evaporated to dryness at reduced pressure to yield the fluorescently labelled PAA. The amount of fluorescent label content was AmNS = $1.55 \mathrm{~mol} \%$ and $\mathrm{ACE}=1.28 \mathrm{~mol} \%$. The labelled polymers were characterised by gel permeation chromatography with a refractive index detector to have a molar mass distribution of: number average molar mass $M_{\mathrm{n}}=19580 \mathrm{~g} \mathrm{~mol}^{-1}$; weight average molar 
mass $M_{\mathrm{W}}=47458 \mathrm{~g} \mathrm{~mol}^{-1}$ and viscosity average molar mass $M_{\mathrm{v}}=$ $37778 \mathrm{~g} \mathrm{~mol}^{-1}$ (the estimated accuracy of $M_{\mathrm{n}}$, and $M_{\mathrm{v}}$ is $\pm 5 \%$ ).

\subsection{Adsorption of poly acrylic acid to calcite}

The adsorption of PAA to $\mathrm{CaCO}_{3}$ was performed using batch type experiments at $\mathrm{pH} 7$ and 11 , at concentrations of $0-5 \mathrm{mM}$ of PAA. An aliquot of $10 \mathrm{~mL}$ of the polymer solution was placed in a $20 \mathrm{~mL}$ centrifuge tube and $1 \mathrm{mg}$ of the calcite powder was added slowly while being stirred and the suspensions were then stirred overnight. After equilibration, samples were centrifuged at $5000 \mathrm{rpm}$ for 40 minutes. The supernatant solution was removed and centrifuged at $5000 \mathrm{rpm}$ for another 40 minutes. Aliquots of the supernatant were carefully removed before and after centrifuging and analysed using fluorescence spectroscopy.

\subsection{Steady state fluorescence measurements}

Fluorescence emission spectra were obtained using a PerkinElmer LS50b spectrometer and a Horiba FluoroMax-4 spectrofluorometer. The excitation wavelength $\left(\lambda_{\text {ex }}\right)$ was $370 \mathrm{~nm}$ and emission was sampled between 380 and $600 \mathrm{~nm}$ at $20{ }^{\circ} \mathrm{C}$ with slit widths of $2.5 \mathrm{~nm}$ for excitation and emission. Fluorescence steady state analyses were performed on the AmNS-labelled PAA calcite solutions before and after separation (via centrifuging). The percentage of the adsorbed amount of PAA was determined by subtracting the fluorescence intensity observed after centrifuging from that determined before separation.

\subsection{Time-resolved fluorescence measurements}

Fluorescence anisotropy data were acquired using an Edinburgh Instruments 199 spectrometer operating on the time-correlated single photon counting principle. An IBH Horiba Jobin Yvon diode based megahertz nanoled (N 370) was used as the excitation source with a full width half maximum pulse width of $800 \mathrm{ps}$ and peak response at $370 \mathrm{~nm}$. The total response time of the detection channel which included a fast photomultiplier (Hamamatsu R3235) was $c a$. 1.2 ns. The instrument was modified through the addition of a second detection channel to allow time-resolved anisotropy measurements (TRAMS) to be made. This channel was equipped with a 'toggling' device, which periodically switched the plane of polarization of the analyser and simultaneously addressed the detector signals to a designated memory segment in the multichannel analyser. This allowed accumulation of the parallel $\left[i_{||}(t)\right]$ and perpendicular $\left[i_{\perp}(t)\right]$ components. An interference filter was used to isolate fluorescence at $450 \mathrm{~nm}$ which resulted in a $\mathrm{G}$ factor of unity in experiments involving the current instrument. Full details of the TRAMS experiments and associated analytical procedures have been described elsewhere. ${ }^{30}$

\subsection{Simulation protocols}

An atactic PAA chain consisting of 28 monomer units was used for the simulations. To increase the sampling of the PAA chain and speed up the simulations, a metadynamics method ${ }^{20}$ was implemented. With metadynamics the trajectory of a system is biased by a history-dependent potential, driving the system to explore a larger conformational space than standard molecular dynamics simulations. The biased potential is created in the form of gaussians added along the trajectory following a set of Collective Variables (CVs). The time evolution of the sum of the added gaussians can be reconstructed in the form of a free energy surface as a function of the chosen CVs.

To sample the conformational space of the polymer, its radius of gyration was used as the $\mathrm{CV}$ for the metadynamics simulations. The radius of gyration is the average distance from the centre of mass of the polymer to each individual monomer and describes the size and shape of the polymer. The radius of gyration is taken as an ensemble average and can be written as

$$
R_{\mathrm{gyr}}=\left(\frac{\sum_{i}^{n} m_{i}\left|r_{i}-r_{\mathrm{cm}}\right|^{2}}{\sum_{i}^{n} m_{i}}\right)^{\frac{1}{2}}
$$

where $n$ is the number of atoms in the polymer, $r_{i}$ is the three dimensional position of atom $i, m_{i}$ is the mass of atom $i$ and $r_{\mathrm{cm}}$ is the centre of mass of the polymer and is defined as

$$
r_{\mathrm{cm}}=\frac{\sum_{i}^{n} r_{i} m_{i}}{\sum_{i}^{n} m_{i}}
$$

The polymer was inserted into a periodic box of 40000 TIP3P water molecules, ${ }^{31}$ packed together randomly using the Packmol code, ${ }^{32}$ and placed on top of a slab of (10.4) oriented calcite with a surface area of $104 \times 108 \AA$ and depth of $32 \AA$ (chosen to ensure a density of water of $0.99 \mathrm{~g} \mathrm{~cm}^{-3}$ ). At pH 6.5 and 9.5 the PAA will have a differing degree of ionisation of the acid groups. The ionisation process cannot be directly modelled in standard classical simulations and therefore it is necessary to set the molecule with the appropriate ionisation at the beginning of the simulation. This was taken from experimental titration data $^{21}$ which indicated ionisation degrees of 0.75 and 1.0 at $\mathrm{pH}$ values of 6.5 and 9.5 respectively. Therefore for the polymer at pH 6.5, 20 side chains were ionised and distributed randomly throughout the polymer to spread out the charge. For the polymer at $\mathrm{pH} 9.5$ all side chains were ionised. All atomic charges were calculated using AMBER Antechamber ${ }^{33}$ using the AM1-BCC method, ${ }^{34}$ a semi-empirical method that calculates the electronic structure in combination with bond charge corrections. To ensure a neutral simulation box 10 and $14 \mathrm{Ca}^{2+}$ cations were added to the simulation cells for $\mathrm{pH} 6.5$ and 9.5 respectively. The dissolution of calcite gives $\mathrm{Ca}^{2+}$ ions to the solution that can form PAA-Ca complexes in solution. Due to the relatively short length of the simulations and the potentially sizable energetic barriers it is entirely possible that the $\mathrm{Ca}^{2+}$ cations will not displace to or from the PAA molecule i.e. they are likely to remain complexed if they begin the simulation complexed and non-complexed if they begin the simulation non-complexed. Over the experimental timeframe we would expect the cations to reach the more stable (lower energy) arrangement. To account for this we ran two sets of simulations. In the first the $\mathrm{Ca}^{2+}$ cations were randomly placed within $<5 \AA$ of the PAA molecule (complexed) and in the second they were placed randomly $>10 \AA$ away from the PAA 
(non-complexed). The polymer was placed either close to the surface of calcite ( $<3 \AA$ - close) or further away from the surface (>15 $\AA$ - far) to simulate the polymer being adsorbed on the surface or in solution. Collectively this made a total of eight different simulations ( $\mathrm{pH}$ 6.5-close-complexed, $\mathrm{pH}$ 6.5-close-noncomplexed, pH 6.5-far-complexed, pH 6.5-far-non-complexed, $\mathrm{pH}$ 9.5-close-complexed, $\mathrm{pH}$ 9.5-close-non-complexed, $\mathrm{pH}$ 9.5-farcomplex, pH 9.5-far-non-complexed).

The Molecular Dynamics package DL POLY Classic ${ }^{35}$ was used for all simulations, with the plug-in Plumed ${ }^{36}$ for the metadynamics simulations. The forcefield for the mineral phase was described by the $\mathrm{CaCO}_{3}$ potentials developed by Raiteri et al. ${ }^{37}$ and the interactions of the organic molecules described with the AMBER forcefield. ${ }^{38}$ To model the interactions between the mineral and organic phase a generic method ${ }^{39}$ to produce cross term potentials was used to generate a reliable potential for our system without the need for fitting the interaction terms. All the potential cross term values are listed in the ESI. $\dagger$ All systems were equilibrated for $1.0 \mathrm{~ns}$ with a timestep of $0.1 \mathrm{fs}$, using an $N V T$ ensemble with a Nosé-Hoover thermostat ${ }^{40}$ with a relaxation time of $0.5 \mathrm{ps}$ and a temperature of $295 \mathrm{~K}$. Subsequent metadynamics simulations were performed for $2.0 \mathrm{~ns}$, and gaussians of height $0.05 \mathrm{~kJ} \mathrm{~mol}^{-1}$ and width 0.35 were added every 0.5 ps. All 2.0 ns of the simulation time was used to collect data to reconstruct the free energy surfaces. To calculate the adsorption energies of the polymer, the lowest energy configuration from each configuration was simulated using Molecular Dynamics simulation without metadynamics, in an NVT ensemble with a timestep of 0.1 fs and a Nosé-Hoover thermostat with a relaxation time of 0.5 ps. These simulations were equilibrated for $0.5 \mathrm{~ns}$, followed by a simulation of $1.0 \mathrm{~ns}$ for data collection.

\subsection{Simulation binding energies}

The configurational adsorption energies ( $\left.E_{\text {adsorption }}\right)$ of the PAA chains onto the surface of calcite are calculated with respect to the water-solvated calcite slab $\left(E_{\text {water-calcite }}\right)$ and water-solvated polymer simulations ( $\left.E_{\text {water-polymer }}\right)$

$E_{\text {adsorption }}=E_{\text {water-polymer-calcite }}-E_{\text {water-calcite }}-E_{\text {water-polymer }}$

where $E_{\text {water-polymer-calcite }}$ is the total energy of the system where the polymer is adsorbed onto the surface of calcite.

\section{Results and discussion}

\subsection{Poly acrylic acid in solution}

The PAA conformation in solution was obtained using two different fluorescent labels. If the fluorophore is covalently attached to a polymer either during or after synthesis then information concerning macromolecular conformations can be obtained via TRAMS and the resultant correlation time, $\tau_{\mathrm{c}}$. For example, a short $\tau_{\mathrm{c}}$ would be associated with an expanded flexible chain while a large $\tau_{\mathrm{c}}$ would be consistent with a collapsed slow moving globular structure. Previous work ${ }^{15,30}$ using an ACE fluorescence label has revealed that PAA undergoes a slight conformational transition from an expanded chain

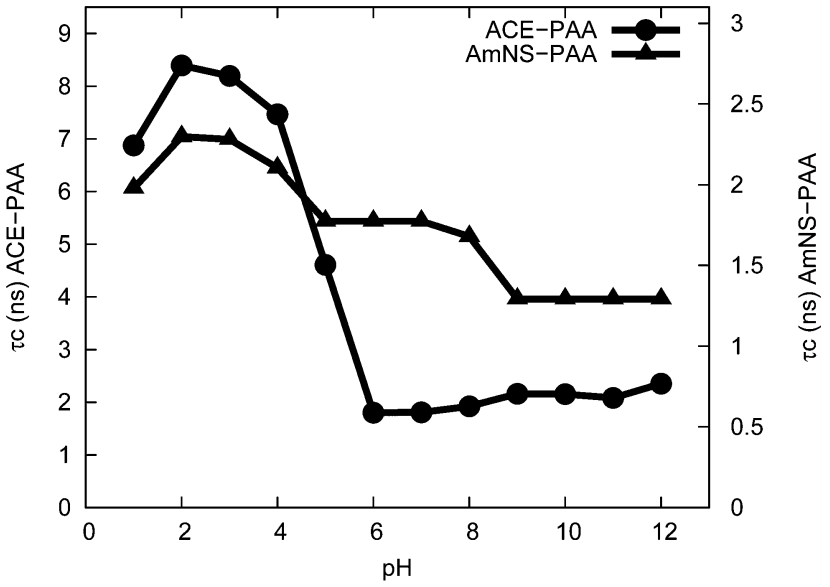

Fig. 1 Correlation time for PAA labelled with AmNS and ACE in solution as a function of $\mathrm{pH}$, measured using time resolved anisotropy measurements (TRAMS).

at high $\mathrm{pH}$, due to carboxylate anion charge repulsions, to a partially collapsed conformation at low degrees of ionisation.

In this work, AmNS- and ACE-labelled PAA were investigated at various $\mathrm{pH}$ values between 1 and 12 using TRAMS. Anisotropy decay data for each $\mathrm{pH}$ was modelled using a single exponential function as detailed in the ESI. $\dagger$ The resultant $\tau_{\mathrm{c}}$ data are shown as a function of $\mathrm{pH}$ in Fig. 1. The AmNS data can be contrasted with the ACE data and with previous anisotropy studies of fluorescence labelled PAA: ${ }^{15}$ clearly the conformational transition for the AmNS-labelled PAA sample is very slight compared to that of the ACE-labelled polymer. The $\tau_{\mathrm{c}}$ for AmNS-labelled PAA at low $\mathrm{pH}$ is $c a .2 .3 \mathrm{~ns}$ compared to $c a$. $8.5 \mathrm{~ns}$ for the ACE-labelled polymer. Presumably this difference stems from the mode of attachment of each label to the polymer and the resultant motions that the respective label can monitor. The ACE label is attached at two points to the polymer backbone and so can only report on macromolecular backbone motion. With the AmNS label, on the other hand, backbone motion is not being exclusively monitored in the current experiments: free rotation about the bond of attachment to the polymer is possible which is reflected in a much shorter $\tau_{\mathrm{c}}$. Nonetheless, this label is still of value in revealing the conformational behaviour of PAA since it is likely to be sensitive to the microviscosity of its environment. Previous work ${ }^{16,41}$ has revealed that various fluorescent labels attached to poly(methacrylic acid) were sensitive to the conformational switch of the polymer through changes in the microviscosity of the medium. The AmNS label on PAA rotates freely at $\mathrm{pH}$ values above 8 due to the fact that the polymer is in its extended conformation. Below pH 6, the polymer contracts into its partially coiled structure. Under these conditions of macromolecular crowding, the microviscosity is increased slightly which results in a decrease of the free rotation of the label, reflected in a slightly longer $\tau_{\mathrm{c}}$. The $\tau_{\mathrm{c}}$ data from AmNS-PAA are, in effect, an indirect measure of the conformational change of PAA. The current anisotropy results also confirm the findings of Laguecir et al. ${ }^{21}$ who studied a PAA chain of molecular weight = 50000 Da with a Rhodamine 123 fluorescence tag in fluorescence 
correlation spectroscopy experiments. The decrease in correlation time observed in the current experiments is consistent with polymer expansion at high $\mathrm{pH}$, and faster motion through the solution. This change from a coiled conformation with a small radius of gyration to a more extended conformation is also visible in the computational simulations of PAA.

Throughout the simulations where the polymer is complexed by counter ions, the $\mathrm{Ca}^{2+}$ ions that are bound to the carboxylate groups of the polymer stay bound and do not tend to dissociate. In the simulations where the polymer is not complexed, the counter ions are further away $(>10 \AA)$ and do not associate at all with the polymer during the simulation time. Therefore the metadynamics sampling of the polymer does not affect the degree of complexation. The complexation of PAA in solution is an energetically favourable reaction when the conformational energies of both systems are compared. The energy difference when complexing the polymer is $-23.7 \pm 16.6 \mathrm{~kJ} \mathrm{~mol}^{-1}$ (pH 6.5) and $-37.5 \pm 20.9 \mathrm{~kJ} \mathrm{~mol}^{-1}$ ( $\left.\mathrm{pH} 9.5\right)$. Therefore we would expect the polymer to be complexed throughout the experiments. We continue to discuss all eight different scenarios, however, as the complexation could vary depending on the presence of the surface and the solution conditions.

The free energy surface of the polymer in solution (Fig. 2), generated with metadynamics simulations demonstrates the influence of $\mathrm{Ca}^{2+}$ counter ions present in solution on the conformation of the polymer in the energetically preferred complexed system. At higher $\mathrm{pH}$ values, above the point of zero charge of carboxylate groups in the molecule, the net surface charge of the polymer is expected to be negative and the acidic groups mainly deprotonated on all the experiments performed. In the vicinity of $\mathrm{Ca}^{2+}$ ions, the polymer adopts an open and extended conformation as shown by the obtained radius of gyration values for the simulations conducted at initial $\mathrm{pH}$ of 6.5 and 9.5 respectively (labelled as complexed). The strong binding of the $\mathrm{Ca}^{2+}$ ions to the carboxylate groups of the polymer induces a local rigidity in the structure, causing the chain to be less flexible. Similar results were seen by Bulo et $a l^{22}$ with similar simulations of $\mathrm{Ca}^{2+}$ ions and PAA chains in water.

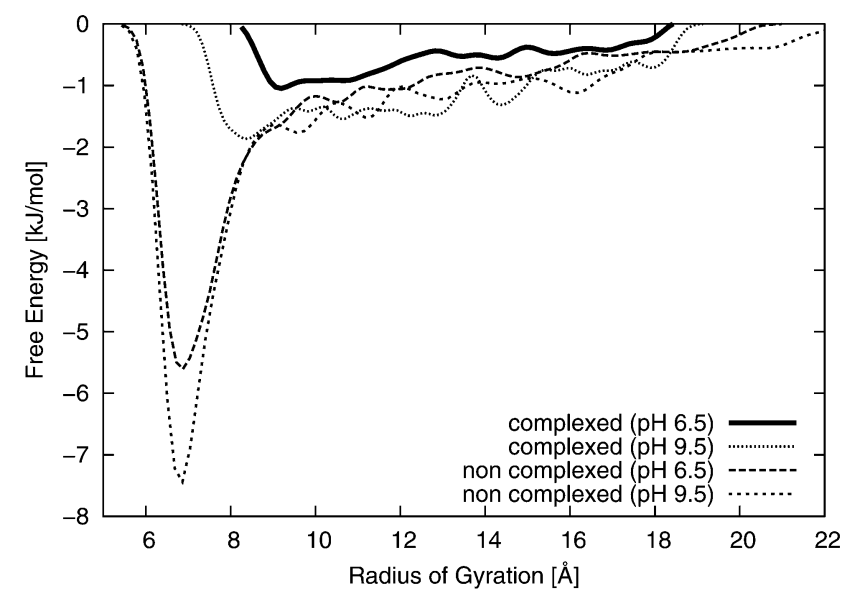

Fig. 2 Free energy surface of the radius of gyration of the polymer in solution, as calculated with the metadynamics simulations.
The removal of $\mathrm{Ca}^{2+}$ ions from solution forces the polymer to contract and adopt a random-coiled configuration, evident from the small radius of gyration and the deep well in the energy surface of $6 \mathrm{~kJ} \mathrm{~mol}^{-1}$ obtained for the non-complexed simulations. The configuration of the polymer in solution is thus greatly influenced by the presence of positively charged ions that impart rigidity to the polymer that is supported by the high density of charges on the polymer backbone.

\subsection{Poly acrylic acid on calcite surfaces}

Experimental data for AmNS-labelled PAA on the surface of calcite has been produced using TRAMS measurements, as seen in Fig. 3. When adsorbing PAA onto the surface of calcite the correlation time increases at $\mathrm{pH} 7$ and 11. In previous studies ${ }^{17}$ of polymer adsorption at surfaces a short $\tau_{\mathrm{c}}$ (ca. $2 \mathrm{~ns}$ ) was reported for a fluorescence-labelled polydimethylacrylamide sample which was free in solution. Upon binding to a silica surface this polymer adsorbed in a train-like conformation and an extremely long $\tau_{\mathrm{c}}(\mathrm{ca} .1 \mu \mathrm{s})$ was observed. In the current study, the increase in correlation time indicates a smaller radius of gyration for the polymer and would indicate that PAA forms a partially coiled structure on the surface of calcite. However, these data may also reflect the fact that motion of the macromolecular chain is restricted as adsorption proceeds and PAA binds to the mineral. At $1.0 \mathrm{wt} \%$ added calcite, the correlation time decreases for both $\mathrm{pH}$ values. This drop off in correlation time (or increase in radius of gyration of the polymer) can be explained by the fact that the surface of the calcite is saturated by a full layer of PAA chains. Additional polymer chains added to the solution cannot adsorb to the surface and are either floating in solution or are loosely bound to other polymer chains in the first adsorption layer. The same effect can be observed when analysing the absorbed amount of PAA onto the calcite surface using steady state fluorescence spectroscopy (Fig. 4) where the amount of adsorbed PAA on calcite reaches a plateau around $3 \mathrm{mM}$ concentration of PAA and remains unchanged at increasing concentrations of PAA in solution. Previous computational studies ${ }^{42}$ have shown a similar effect, where $\mathrm{Ca}^{2+}$ ions associate with single PAA chains, shielding the negative

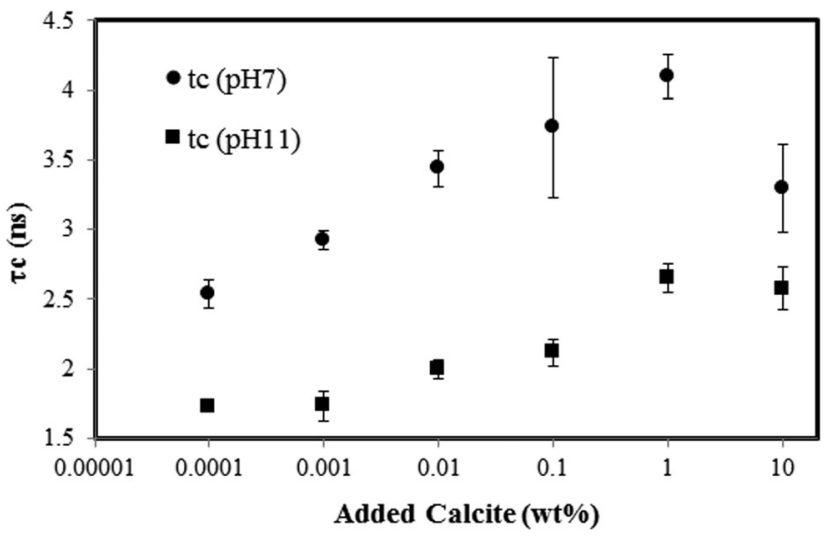

Fig. 3 Correlation time for PAA labelled with AmNS on calcite surface, measured using time resolved anisotropy measurements (TRAMS). 


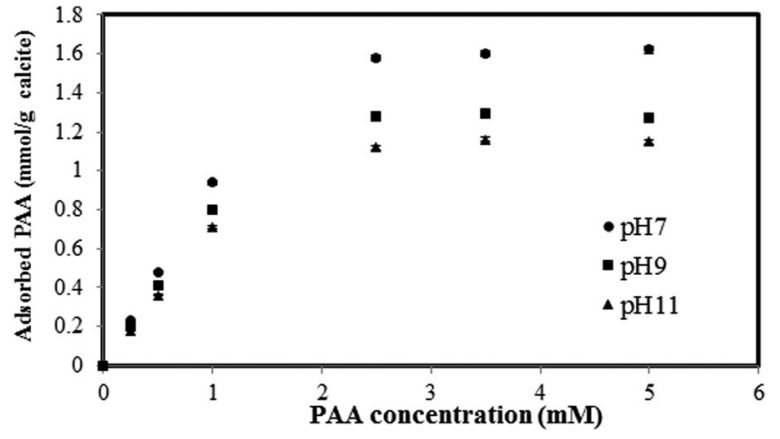

Fig. 4 Sorption isotherm of PAA on calcite surface at pH 7, 9 and 11 quantified using steady state fluorescence spectroscopy.

charges on the polymer chains and facilitating the agglomeration of polymers on the mineral surface to form biofilms.

The sorption of a polyacrylate to calcite has been previously reported ${ }^{43-45}$ to follow a similar trend to the one observed here and the general model of sorption of electrolyte on oxide. ${ }^{45}$ At low polyacrylate concentration in solution a monolayer of polymer is formed on the surface of calcite until saturation is reached at higher concentration of polymer in solution. ${ }^{43,44}$ The amount of polyacrylate sorbed to the surface of calcite is affected by the $\mathrm{pH}$ of the solution, where more polyacrylate is sorbed at $\mathrm{pH}$ values lower than the $\mathrm{pH}_{\mathrm{pzc}}$ of calcite. The reduction of sorption of polyacrylate and/or PAA to calcite at $\mathrm{pH}$ values higher than 7 indicates a change in binding mechanism from simple electrostatic interactions between molecule and surface, since PAA would be negatively charged and calcite would have an overall positive charge producing strong binding. At $\mathrm{pH}<\mathrm{pH}_{\mathrm{pzc}-\text { calcite }}$ the PAA chains are negatively charged due to the deprotonation of carboxyl groups of PAA resulting in the ionization of PAA. ${ }^{21}$ The degree of ionization increases with increasing $\mathrm{pH}$ to values above the $\mathrm{pH}_{\mathrm{pzc}-\text { calcite }}$ resulting in an overall negative charge on the surface of polyacrylic acid. The degree of ionization for PAA of $M_{\mathrm{w}}=50000 \mathrm{~g} \mathrm{~mol}^{-1}$ (similar to the one used in this study) at $\mathrm{pH} 7$ was reported to be $\alpha=0.55$, suggesting that more than $50 \%$ of the carboxyl groups on the PAA are negatively charged. At $\mathrm{pH} 11, \alpha=1.0$ and therefore PAA is fully ionized in solution. ${ }^{21}$ This suggests that the sorption of PAA to calcite is favourable at $\mathrm{pH} 7$, as observed from the sorption isotherm obtained.

The metadynamics simulations of the PAA chain on the surface of calcite show a similar, but less pronounced behaviour than the polymer in solution. The radius of gyration of the macromolecule is dependent on both the $\mathrm{pH}$ of the solution and the presence of counter ions. The free energy of the polymer on the surface of calcite as a function of the radius of gyration is shown in Fig. 5. The relatively strong interaction between the surface of calcite and the short chain polymer prevents the latter from desorbing from the surface during the metadynamics simulations. This surface binding also has an effect on the complexation of the $\mathrm{Ca}^{2+}$ counter ions, which have less effect on the configuration than in solution, as the interaction between the calcite surface and the polymer dominates.

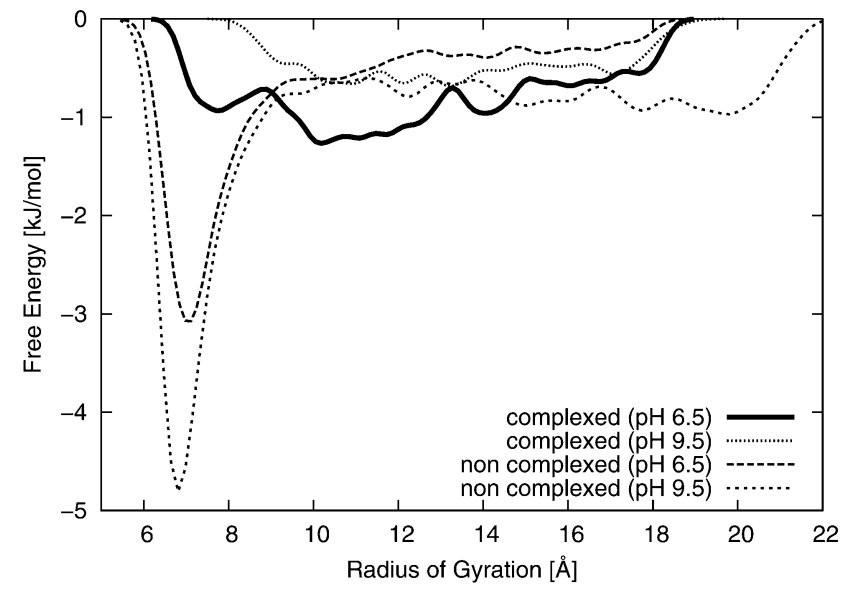

Fig. 5 Free energy surface of the radius of gyration of the polymer on the surface of calcite solution, as calculated with the metadynamics simulations.

The adsorption free energy $(\Delta G)$ of the PAA adsorption process on the calcite surfaces can be calculated using the equilibrium constant obtained from the isotherm fitted to the data in Fig. 4. A Langmuir isotherm model was fitted to the data to produce adsorption gibbs free energies of $-2.12,-1.41$ and $-1.18 \mathrm{~kJ} \mathrm{~mol}^{-1}$ at $\mathrm{pH}$ 7, 9 and 11 respectively. The negative values for $\Delta G$ indicates a favourable binding of PAA to the surface of calcite, with the polymer binding more strongly to the surface at lower $\mathrm{pH}$ values, when less functional groups are ionised. These experimental adsorption energies are within the same range of those obtained by Geffroy et al., ${ }^{26}$ who measured an adsorption enthalpy of $+2 \mathrm{~kJ} \mathrm{~mol}^{-1}$ for PAA adsorption onto calcite.

The simulations show that binding occurs through several of the acid groups of the polymer which interact with the calcite surface. A wide range of adsorption energies of PAA on calcite have been obtained from previous simulations. Using a PAA dimer in a simulation with a TIP3P water model and a flat (104) calcite surface, Zhu et al. ${ }^{14}$ calculated an adsorption energy of $-45 \mathrm{~kJ} \mathrm{~mol}^{-1}$ for a dimer where both carboxyl groups interact with the surface mediated by water. On the other hand, Aschauer et $a l^{25}$ obtained a positive binding energy of $+395 \mathrm{~kJ} \mathrm{~mol}^{-1}$ for simulations of a 10 monomer PAA chain on acute stepped calcite. The authors offer two possible explanations for this surprising result: the presence of a strongly layered water layer that keeps the PAA molecule at a distance from the surface (ca. $0.4 \mathrm{~nm}$ ) and formation of complexes which stabilise the PAA in a coiled configuration in solution. Our metadynamics simulations do show the existence of complexation effects, but this does not prevent strong binding to the calcite surface. The advantage of using metadynamics over standard molecular dynamics simulations is that it compels the system to sample a wide range of configurations both in the solution and at the surface. This supports the suggestion that barriers resulting from a combination of complexation and strongly layered surface water was preventing the calculations of Aschauer et al. ${ }^{25}$ from finding the low-energy binding state found in our simulations.

When calculating the energy of adsorption of molecules to a mineral surface from the computational simulations, standard 
practice is to calculate the configurational energy of adsorption. This method only takes into account the enthalpic contribution of the system and ignores the entropic contribution to the binding. Adsorption of macromolecules to the surface of calcite will be a positive entropic process, as the binding process will disrupt the highly ordered water layers on the surface of calcite. In order to calculate an estimate of the free energy of adsorption, the entropic energy of the release of these water molecules needs to be taken into account. An estimate of the Helmholtz free energy, $A$, (the free energy without the contribution from the pressure volume term as these simulations are performed within the NVT ensemble) of the system can be made by assuming the most important entropy term arises from the displacement of water molecules from the surface. Given that our metadynamics simulations showed similar conformational behaviour in solution and on the surface, it is reasonable to assume there is a very small entropy change between the polymer on the surface on in solution, and therefore the estimated free energy of the system can be written as $A=U-T n S$, where $U$ is the internal energy of the system, $T$ is the temperature, $n$ is the number of water molecules displaced from the calcite surface during adsorption and $S$ is the entropic contribution arising from the displacement of a single water molecule from the calcite surface. Freeman and Harding ${ }^{46}$ estimated this entropic contribution for a water molecule on the surface of (10.4) calcite to be $c a .6 \mathrm{~J} \mathrm{~mol}^{-1} \mathrm{~K}^{-1}$.

The configurational energy of adsorption and estimated Helmholtz free energy of adsorption are shown in Table 1. The predicted binding energy decreases with increasing $\mathrm{pH}$ values, indicating that the polymer binds more strongly to the calcite surfaces at lower $\mathrm{pH}$ values.

We finally address the issue of the difference between the free energy obtained from a Langmuir isotherm analysis of the experimental absorption curve and the calculated energy of absorption for the PAA molecule. It is clear from the great difference in values that they must refer to different quantities. The free energy value estimated using the Langmuir isotherm constant may include mediated bonding through calcium ions and even a contribution from PAA molecules that are loosely attached to already absorbed PAA molecules that are loosely attached to an absorbed PAA layer. This is supported by the TRAMS data that suggests that the calcite is saturated with PAA chains during the experiment with more than one type of bonding between the PAA molecules and the mineral unlike the simulation where we bind one isolated polymer. It should be pointed out that saturation does not imply that all the binding sites are occupied. Jamming, whereby the molecules block large number of possible sites, is almost inevitable in a system of this kind. ${ }^{47}$ The simulation refers (necessarily) to a smaller molecule than that used in experiment that is in isolation on the surface and the binding functional groups are directly attached to the surface with no intermediate water present between the group and the mineral. This individual binding will give a much larger binding energy than the average response collected in the experiment.

The number of functional groups interacting with the calcite surface upon binding can be calculated from the computational simulations and is displayed in Table 2. The number of displaced water molecules depends strongly on the conformation adopted by the PAA chain, with twice as many water molecules displaced when the polymer adopts an extended conformation than for a coiled conformation. Similarly, the number of functional groups involved with the binding process changes depending on the adopted conformation. In an extended conformation (Fig. 6) the average number of carboxylic acid groups interacting with the surface is 6.9 , corresponding to $25 \%$ of the total number of functional groups on the polymer. This number drops to $4.0(14 \%)$ for a more coiled conformation (Fig. 6). The configurational binding energies per functional group interacting with the surface at $\mathrm{pH} 9.5$ are $-45.7 \mathrm{~kJ} \mathrm{~mol}^{-1}$ (coiled) and $-47.3 \mathrm{~kJ} \mathrm{~mol}^{-1}$ (extended), suggesting that there is a much larger entropic gain with the adsorption of the extended conformation on the surface of calcite than with the coiled conformation.

The values presented in Table 2 show that only up to a quarter of the functional groups present in the polymer are involved with the binding process, and that this number does not drastically increase when increasing the number of charged functional groups when increasing the $\mathrm{pH}$ of the environment. When analysing a typical binding motif of an extended polymer chain on the surface of calcite (e.g. Fig. 6) it can be seen that only the central part of the polymer stays in constant contact with the surface and that the ends of the polymer are often found floating up into the solution. Additionally, only alternating

Table 2 Average number of functional groups of PAA interacting with the calcite surface during the simulations

\begin{tabular}{lllll}
\hline Conformation & pH 6.5 & pH 9.5 & Average & Percentage $^{a}(\%)$ \\
\hline Extended & $7.1 \pm 0.9$ & $6.7 \pm 0.8$ & 6.9 & 25 \\
Coiled & $4.0 \pm 0.6$ & $3.9 \pm 0.7$ & 4.0 & 14
\end{tabular}

${ }^{a}$ Percentage of total number of functional groups.

Table 1 Calculated values for the energy of adsorption of PAA onto the surface of (104) calcite

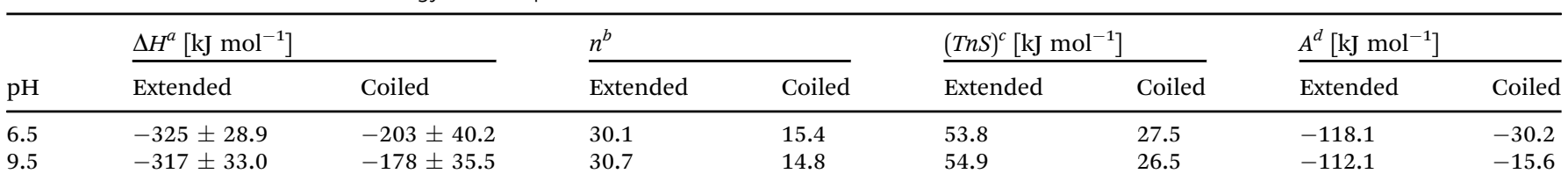

${ }^{a}$ Enthalpy of adsorption. ${ }^{b}$ Number of displaced water molecules. ${ }^{c}$ Entropic contribution due to displaced water molecules. ${ }^{d}$ Estimated free energy of adsorption. 


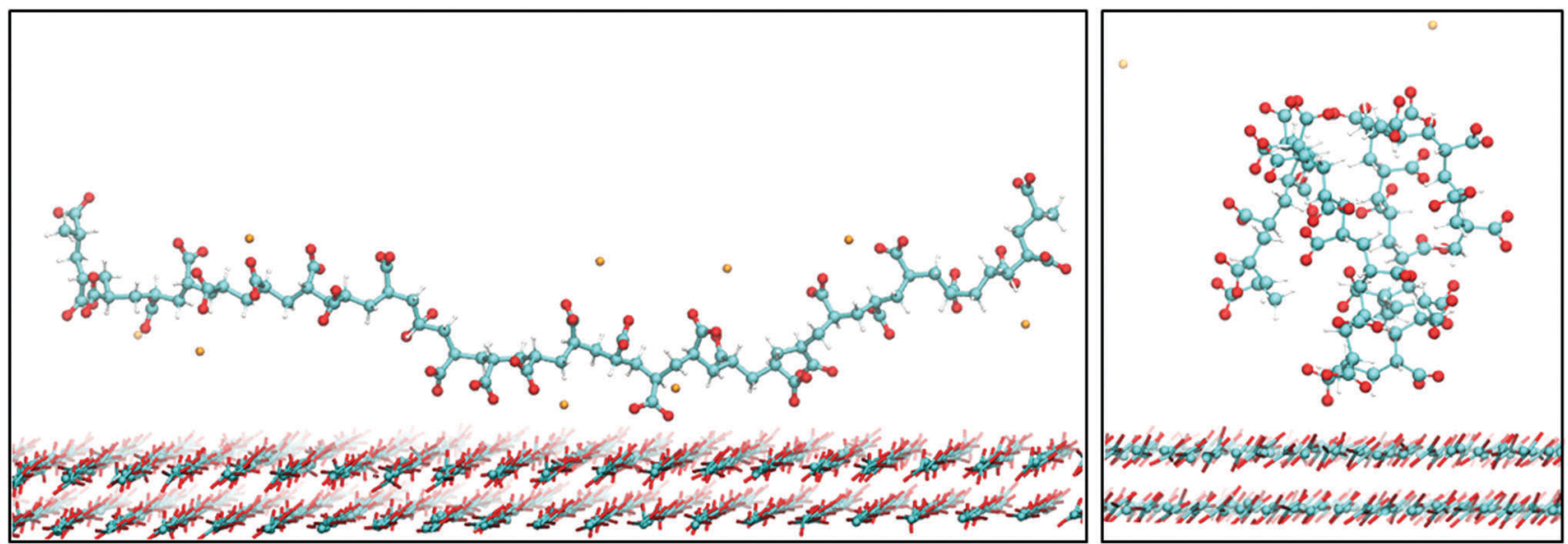

Fig. 6 Typical binding motif of an extended PAA chain (left) and a coiled PAA chain (right) on the surface of (104) calcite. Oxygen, carbon, hydrogen atoms and $\mathrm{Ca}^{2+}$ counter ions are depicted in red, cyan, white and orange respectively. Water molecules are omitted for clarity.

functional groups in the central section of the polymer are involved in the binding process. This behaviour suggests that not only the number of charged functional groups present will dictate the binding of a molecule to surfaces, but also the spacing between these groups. On the surface of (104) calcite there are a lot of exposed $\mathrm{CO}_{3}{ }^{2-}$ ions present, which could hinder the adsorption of molecules with a high density of negative charges. The complexation of the polymer with positively charged calcium ions might therefore be aiding the adsorption of the polymer to the calcite surface by shielding these negative charges. The counter ions are complexed to the polymer for extended periods of time and continue to be associated with the polymer when bound to the surface, indicating this complexation could be a mechanism of calcium transport to the surface.

\section{Conclusions}

In this work we studied the behaviour of PAA in solution and on the surface of calcite using experimental and computational techniques. The role of calcium counter ions in the system is of great importance for the conformation and binding of the polymer. PAA complexed with $\mathrm{Ca}^{2+}$ counter ions will prefer an extended conformation in which the counter ions are strongly associated with the carboxylic acid functional group throughout the simulations, whereas the conformation adopted by the polymer when not complexed by counter ions is a coiled, sphere like structure. The extended conformation has a higher adsorption energy on the calcite surface than the adopted conformation of the polymer when not complexed. The difference in size of the polymer molecule used during the laboratory experiments compared to the computational simulation was rather large and therefore a direct comparison of the binding energies was not possible. Despite this difference, the binding mechanism revealed using both approaches as well as the trends in energy values found are similar, demonstrating that both studies provide direct evidence of the mechanism of interactions between the polymer molecules and the surface of calcite. The long time-scales of the polymer-calcium association, even when binding to the surface of calcite, provides an insight into a possible mechanism of how the calcium ions are transported to the surface of calcite. The computational methodology developed in this work to study the conformation and binding of large macromolecules in solution and on the surface of calcite can be used in future to study similar sized macromolecules. The large differences in adsorption energies between experiment and simulation can be explained by the differences in molecular weight of the polymer used as the short chained polymers are expected to have a higher affinity for the surface. The adsorption experiments performed using steady state fluorescence spectroscopy and the computational simulations of adsorption both indicate that PAA is readily adsorbed onto the surface of calcite due to the functional groups present in the molecule. The strength of this adsorption is dependent on the $\mathrm{pH}$ of the system and the presence of counter ions in solution. In a lower $\mathrm{pH}$ environment, the polymer binds more strongly to the surface of calcite. The enhanced binding of the extended conformation PAA on the surface of calcite shows that flexible chains, such as intrinsically disordered proteins, could be beneficial to the binding of these macromolecules. The fact that only $14-25 \%$ of the functional groups present on the polymer are involved in the binding process suggests that the total number of functional groups is not the only factor that plays a role in the adsorption of biomolecules on the surface of minerals. In addition the total charge, the spacing between these charged groups to match the underlying charge pattern of the mineral surface plays a role, a fact that is important for the future design of biomolecules to interact with these mineral surfaces.

\section{Acknowledgements}

We thank the EPSRC funded consortium "Hard-soft matter interfaces: from understanding to engineering", (EP/I001514/1) for financial support and the provision of computer time on the ARCHER UK National Supercomputing Service (http://www. archer.ac.uk). This Programme Grant funds the Materials Interface with Biology (MIB) consortium. Access to ARCHER was also 
obtained via our membership of the UK's HEC Materials Chemistry Consortium, which is funded by EPSRC (EP/L000202). Further information concerning the underlying data for this work can be obtained by contacting rdm@sheffield.ac.uk and quoting the paper reference.

\section{References}

1 L. Hall-Stoodley, J. W. Costerton and P. Stoodley, Nat. Rev. Microbiol., 2004, 2, 95-108.

2 N. Khardori and M. Yassien, J. Ind. Microbiol., 1995, 15, 141-147.

3 D. R. Elliott, J. D. Scholes, S. F. Thornton, A. Rizoulis, S. A. Banwart and S. A. Rolfe, FEMS Microbiol. Ecol., 2010, 71, 247-259.

4 M. Geoghegan, J. S. Andrews, C. A. Biggs, K. E. Eboigbodin, S. Rolfe, J. Scholes, J. J. Ojeda, M. E. Romero-Gonzalez, R. G. J. Edyvean, L. Swanson, R. Rutkaite, R. Fernando, Y. Pen, Z. Zhang and S. A. Banwart, Faraday Discuss., 2008, 139, 85-103.

5 J. S. Andrews, S. A. Rolfe, W. E. Huang, J. D. Scholes and S. A. Banwart, Environ. Microbiol., 2010, 12, 2496-2507.

6 C. Mayer, R. Moritz, C. Kirschner, W. Borchard, R. Maibaum, J. Wingender and H.-C. Flemming, Int. J. Biol. Macromol., 1999, 26, 3-16.

7 I. W. Kim, S. Collino, D. E. Morse and J. S. Evans, Cryst. Growth Des., 2006, 6, 1078-1082.

8 F. F. Amos and J. S. Evans, Biochemistry, 2009, 48, 1332-1339.

9 C. B. Ponce and J. S. Evans, Cryst. Growth Des., 2011, 11, 4690-4696.

10 S. Elhadj, E. A. Salter, A. Wierzbicki, J. J. De Yoreo, N. Han and P. M. Dove, Cryst. Growth Des., 2006, 6, 197-201.

11 K. Henriksen, S. Stipp, J. Young and M. Marsh, Am. Mineral., 2004, 89, 1709-1716.

12 J. J. J. M. Donners, R. J. M. Nolte and N. A. J. M. Sommerdijk, J. Am. Chem. Soc., 2002, 124, 9700-9701.

13 A. Ghimire, R. M. Kasi and C. V. Kumar, J. Phys. Chem. B, 2014, 118, 5026-5033.

14 B. Zhu, X. Xu and R. Tang, J. Chem. Phys., 2013, 139, 234705.

15 J. Ebdon, B. Hunt, D. Lucas, I. Soutar, L. Swanson and A. Lane, Can. J. Chem., 1995, 73, 1982-1994.

16 I. Soutar and L. Swanson, Macromolecules, 1994, 27, 4304-4311.

17 I. Soutar, L. Swanson, S. Wallace, K. Ghiggino, D. Haines and T. Smith, ACS Symp. Ser., 1995, 363.

18 Y. Sugita and Y. Okamoto, Chem. Phys. Lett., 1999, 314, 141-151.

19 G. M. Torrie and J. P. Valleau, J. Comput. Phys., 1977, 23, 187-199.

20 A. Laio and M. Parrinello, Proc. Natl. Acad. Sci. U. S. A., 2002, 99, 12562-12566.

21 A. Laguecir, S. Ulrich, J. Labille, N. Fatin-Rouge, S. Stoll and J. Buffle, Eur. Polym. J., 2006, 42, 1135-1144.
22 R. E. Bulo, D. Donadio, A. Laio, F. Molnar, J. Rieger and M. Parrinello, Macromolecules, 2007, 40, 3437-3442.

23 G. A. Tribello, C. Liew and M. Parrinello, J. Phys. Chem. B, 2009, 113, 7081-7085.

24 Q. Liu, Q. Wang and L. Xiang, Appl. Surf. Sci., 2008, 254, 7104-7108.

25 U. Aschauer, D. Spagnoli, P. Bowen and S. C. Parker, J. Colloid Interface Sci., 2010, 346, 226-231.

26 C. Geffroy, J. Persello, A. Foissy, B. Cabane and F. Tournilhac, Rev. Inst. Fr. Pet., 1997, 52, 183-190.

27 L. Schultz, M. Andersson, K. N. Dalby, D. Müter, D. Okhrimenko, H. Fordsmand and S. Stipp, J. Cryst. Growth, 2013, 371, 34-38.

28 L. Schultz, L. Dideriksen, S. Lakshtanov, S. Hakim, D. Müter, F. Haußer, K. Bechgaard and S. Stipp, Cryst. Growth Des., 2014, 14, 552-558.

29 D. E. Bergbreiter, G. Tao, J. G. Franchina and L. Sussman, Macromolecules, 2001, 34, 3018-3023.

30 I. Soutar, L. Swanson, R. E. Imhof and G. Rumbles, Macromolecules, 1992, 25, 4399-4405.

31 W. L. Jorgensen, J. Chandrasekhar, J. D. Madura, R. W. Impey and M. L. Klein, J. Chem. Phys., 1983, 79, 926-935.

32 L. Martnez, R. Andrade, E. G. Birgin and J. M. Martnez, J. Comput. Chem., 2009, 30, 2157-2164.

33 J. Wang, W. Wang, P. A. Kollman and D. A. Case, J. Mol. Graphics Modell., 2006, 25, 247-260.

34 A. Jakalian, D. B. Jack and C. I. Bayly, J. Comput. Chem., 2002, 23, 1623-1641.

35 W. Smith and T. Forester, J. Mol. Graphics, 1996, 14, 136-141.

36 M. Bonomi, D. Branduardi, G. Bussi, C. Camilloni, D. Provasi, P. Raiteri, D. Donadio, F. Marinelli, F. Pietrucci and R. A. Broglia, et al., Comput. Phys. Commun., 2009, 180, 1961-1972.

37 P. Raiteri, J. D. Gale, D. Quigley and P. M. Rodger, J. Phys. Chem. C, 2010, 114, 5997-6010.

38 J. Wang, R. M. Wolf, J. W. Caldwell, P. A. Kollman and D. A. Case, J. Comput. Chem., 2004, 25, 1157-1174.

39 C. L. Freeman, J. H. Harding, D. J. Cooke, J. A. Elliott, J. S. Lardge and D. M. Duffy, J. Phys. Chem. C, 2007, 111, 11943-11951.

40 W. G. Hoover, Phys. Rev. A: At., Mol., Opt. Phys., 1985, 31, 1695.

41 L. Ruiz-Pérez, A. Pryke, M. Sommer, G. Battaglia, I. Soutar, L. Swanson and M. Geoghegan, Macromolecules, 2008, 41, 2203-2211.

42 F. Molnar and J. Rieger, Langmuir, 2005, 21, 786-789.

43 J. Chen, T. He, W. Wu, C. Dapeng, J. Yun and C. King Tan, Colloids Surf., A, 2004, 232, 163-168.

44 R. Eriksson, J. Merta and J. Rosenholm, J. Colloid Interface Sci., 2007, 313, 184-193.

45 J. Gebhardt and D. Fuerstenau, Colloids Surf., 1983, 7, 221-231.

46 C. L. Freeman and J. H. Harding, J. Phys. Chem. C, 2014, 118, 1506-1514.

47 M. M. Pradas, M. S. Sánchez, G. G. Ferrer and J. L. G. Ribelles, J. Chem. Phys., 2004, 121, 8524-8531. 